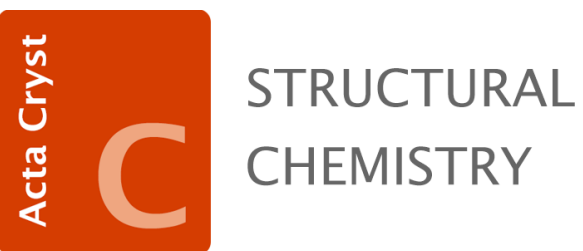

Volume 77 (2021)

Supporting information for article:

Synthesis of luminescent cocrystals based on fluoranthene and the analysis of weak interaction and photophysical properties

Pengfei Wu, Long Zhou, Shuwei Xia and Liangmin Yu 
Table S1. Crystallographic data of the cocrystals

\begin{tabular}{|c|c|c|c|}
\hline Identification code & Fluoranthene-TCNB & Fluoranthene-TFP & Fluoranthene-OFN \\
\hline CCDC code & 2083971 & 2084789 & 2079939 \\
\hline Empirical formula & $\mathrm{C}_{10} \mathrm{H}_{2} \mathrm{~N}_{4} \cdot \mathrm{C}_{16} \mathrm{H}_{10}$ & $\mathrm{C}_{8} \mathrm{~F}_{4} \mathrm{~N}_{2} \cdot \mathrm{C}_{16} \mathrm{H}_{10}$ & $\mathrm{C}_{10} \mathrm{~F}_{8} \cdot \mathrm{C}_{16} \mathrm{H}_{10}$ \\
\hline Formula weight & 380.40 & 402.34 & 474.34 \\
\hline Temperature/K & 170 & 170 & 170 \\
\hline Crystal system & Monoclinic & Monoclinic & Monoclinic \\
\hline Space group & $P 2_{1} / c$ & $P 2_{1} / c$ & $P 2_{1}$ \\
\hline $\mathrm{a} / \mathrm{A}$ & $7.3192(14)$ & $15.4617(12)$ & $6.773(1)$ \\
\hline $\mathrm{b} / \AA$ & $8.2802(8)$ & $7.0577(6)$ & $14.323(2)$ \\
\hline $\mathrm{c} / \AA ̊ \AA$ & $31.3093(4)$ & $16.9113(16)$ & $10.0200(14)$ \\
\hline$\alpha /^{\circ}$ & 90 & 90 & 90 \\
\hline$\beta /{ }^{\circ}$ & 90.162 & 95.095 & 99.732 \\
\hline$\gamma /{ }^{\circ}$ & 90 & 90 & 90 \\
\hline Volume $/ \AA^{3}$ & 1897.5 & 1838.1 & $958.0(2)$ \\
\hline $\mathrm{Z}$ & 4 & 4 & 2 \\
\hline
\end{tabular}


Table S2 Mulliken charges of the dimers

\begin{tabular}{|c|c|c|c|c|c|c|c|c|c|c|c|}
\hline \multicolumn{4}{|c|}{ Fluoranthene-TCNB } & \multicolumn{4}{|c|}{ Fluoranthene-TFP } & \multicolumn{4}{|c|}{ Fluoranthene-OFN } \\
\hline \multicolumn{2}{|c|}{ Fluoranthene } & \multicolumn{2}{|c|}{ TCNB } & \multicolumn{2}{|c|}{ Fluoranthene } & \multicolumn{2}{|c|}{ TFP } & \multicolumn{2}{|c|}{ Fluoranthene } & \multicolumn{2}{|c|}{ OFN } \\
\hline $\mathrm{C} 7$ & - & N1 & - & $\mathrm{C} 1$ & 0.038 & F3 & - & $\mathrm{C} 8$ & 0.291 & $\mathrm{~F} 1$ & - \\
\hline & 0.524 & & 0.349 & & & & 0.316 & & & & 0.137 \\
\hline \multirow[t]{2}{*}{$\mathrm{C} 8$} & - & $\mathrm{N} 2$ & - & $\mathrm{H} 2$ & 0.128 & $\mathrm{~F} 4$ & - & C9 & 0.115 & $\mathrm{~F} 2$ & - \\
\hline & 0.025 & & 0.352 & & & & 0.319 & & & & 0.136 \\
\hline \multirow[t]{2}{*}{$\mathrm{C} 9$} & - & $\mathrm{C} 3$ & 0.672 & $\mathrm{C} 5$ & - & $\mathrm{F} 21$ & - & $\mathrm{C} 11$ & 0.247 & F3 & - \\
\hline & 0.028 & & & & 0.230 & & 0.313 & & & & 0.120 \\
\hline \multirow[t]{2}{*}{$\mathrm{C} 11$} & - & $\mathrm{C} 4$ & - & H6 & 0.128 & $\mathrm{~F} 22$ & - & $\mathrm{C} 13$ & - & $\mathrm{F} 4$ & - \\
\hline & 0.062 & & 0.701 & & & & 0.318 & & 0.441 & & 0.135 \\
\hline \multirow[t]{2}{*}{$\mathrm{C} 14$} & - & $\mathrm{C} 5$ & 0.684 & $\mathrm{C} 7$ & 0.045 & $\mathrm{~N} 23$ & - & $\mathrm{H} 14$ & 0.189 & F5 & - \\
\hline & 0.009 & & & & & & 0.335 & & & & 0.121 \\
\hline \multirow[t]{2}{*}{$\mathrm{C} 15$} & - & C6 & - & $\mathrm{H} 8$ & 0.126 & $\mathrm{~N} 24$ & - & $\mathrm{C} 17$ & - & F6 & - \\
\hline & 0.385 & & 0.679 & & & & 0.351 & & 0.184 & & 0.135 \\
\hline C19 & 0.095 & $\mathrm{C} 10$ & 0.614 & H9 & 0.128 & $\mathrm{C} 25$ & 1.168 & H18 & 0.159 & C7 & 0.370 \\
\hline \multirow[t]{2}{*}{$\mathrm{H} 20$} & 0.128 & N12 & - & $\mathrm{C} 10$ & 0.064 & $\mathrm{C} 26$ & 0.157 & C19 & 0.246 & $\mathrm{C} 10$ & 0.072 \\
\hline & & & 0.359 & & & & & & & & \\
\hline \multirow[t]{2}{*}{$\mathrm{C} 21$} & 0.082 & $\mathrm{C} 13$ & 0.535 & $\mathrm{C} 11$ & 0.111 & $\mathrm{C} 28$ & 0.174 & $\mathrm{C} 20$ & 0.320 & $\mathrm{C} 12$ & - \\
\hline & & & & & & & & & & & 0.050 \\
\hline \multirow[t]{2}{*}{$\mathrm{H} 22$} & 0.121 & $\mathrm{C} 16$ & 0.541 & $\mathrm{C} 12$ & 0.013 & $\mathrm{C} 29$ & - & $\mathrm{C} 22$ & - & C15 & 0.026 \\
\hline & & & & & & & 0.578 & & 0.282 & & \\
\hline $\mathrm{C} 23$ & 0.120 & H17 & 0.180 & $\mathrm{C} 13$ & 0.102 & $\mathrm{C} 30$ & 1.043 & $\mathrm{H} 23$ & 0.167 & $\mathrm{C} 16$ & 0.361 \\
\hline \multirow[t]{2}{*}{$\mathrm{H} 24$} & 0.130 & $\mathrm{C} 18$ & - & H14 & 0.125 & C31 & 0.245 & $\mathrm{C} 24$ & - & $\mathrm{C} 21$ & 0.028 \\
\hline & & & 0.677 & & & & & & 0.443 & & \\
\hline \multirow[t]{2}{*}{$\mathrm{C} 25$} & - & N33 & - & $\mathrm{C} 15$ & 0.038 & C32 & 0.185 & $\mathrm{H} 25$ & 0.189 & $\mathrm{C} 27$ & 0.072 \\
\hline & 0.058 & & 0.379 & & & & & & & & \\
\hline \multirow[t]{2}{*}{$\mathrm{H} 26$} & 0.119 & C34 & 0.535 & $\mathrm{C} 16$ & - & C33 & - & $\mathrm{C} 26$ & 0.287 & C30 & - \\
\hline & & & & & 0.165 & & 0.525 & & & & 0.050 \\
\hline
\end{tabular}




\begin{tabular}{|c|c|c|c|c|c|c|c|c|c|c|}
\hline \multirow[t]{2}{*}{$\mathrm{C} 27$} & - & H35 & 0.185 & C17 & 0.156 & - & $\mathrm{C} 28$ & - & F33 & - \\
\hline & 0.163 & & & & & 0.083 & & 0.281 & & 0.127 \\
\hline \multirow[t]{2}{*}{$\mathrm{H} 28$} & 0.129 & C36 & - & H18 & 0.123 & & $\mathrm{H} 29$ & 0.167 & F34 & - \\
\hline & & & 0.542 & & & & & & & 0.127 \\
\hline \multirow[t]{2}{*}{ C29 } & - & & & C19 & - & & C31 & - & C37 & 0.108 \\
\hline & 0.139 & & & & 0.237 & & & 0.184 & & \\
\hline H30 & 0.124 & & & $\mathrm{H} 20$ & 0.128 & & H32 & 0.158 & C38 & 0.109 \\
\hline \multirow[t]{2}{*}{ C31 } & - & & & $\mathrm{C} 27$ & - & & C35 & - & & 0.008 \\
\hline & 0.085 & & & & 0.581 & & & 0.192 & & \\
\hline \multirow[t]{2}{*}{ H32 } & 0.130 & & & C34 & - & & H36 & 0.159 & & \\
\hline & & & & & 0.364 & & & & & \\
\hline \multirow[t]{2}{*}{ C37 } & 0.052 & & & C35 & - & & C39 & - & & \\
\hline & & & & & 0.083 & & & 0.507 & & \\
\hline H38 & 0.119 & & & H36 & 0.128 & & $\mathrm{H} 40$ & 0.178 & & \\
\hline \multirow[t]{2}{*}{ C39 } & 0.114 & & & C37 & - & & C41 & - & & \\
\hline & & & & & 0.002 & & & 0.507 & & \\
\hline $\mathrm{H} 40$ & 0.130 & & & H38 & 0.126 & & $\mathrm{H} 42$ & 0.178 & & \\
\hline \multirow[t]{2}{*}{ C41 } & - & & & C39 & - & & $\mathrm{C} 43$ & - & & \\
\hline & 0.152 & & & & 0.089 & & & 0.196 & & \\
\hline $\mathrm{H} 42$ & 0.129 & & & $\mathrm{H} 40$ & 0.127 & & $\mathrm{H} 44$ & 0.159 & & \\
\hline \multicolumn{2}{|c|}{+0.092} & \multicolumn{2}{|c|}{-0.092} & \multicolumn{2}{|c|}{+0.083} & -0.083 & \multicolumn{2}{|c|}{-0.008} & \multicolumn{2}{|c|}{+0.008} \\
\hline
\end{tabular}


Table S3. Topological parameters of the cocrystals

\begin{tabular}{|c|c|c|c|c|c|c|c|c|}
\hline $\begin{array}{l}\text { Hydrogen } \\
\text { bonding }\end{array}$ & $\mathrm{r}(\AA)$ & $\rho$ (a.u.) & $\begin{array}{c}\nabla^{2} \rho \\
\text { (a.u.) }\end{array}$ & $\begin{array}{c}\mathrm{G} \\
\text { (a.u.) }\end{array}$ & $\begin{array}{c}\mathrm{V} \\
\text { (a.u.) }\end{array}$ & $\begin{array}{c}\mathrm{H} \\
\text { (a.u.) }\end{array}$ & $\begin{array}{c}E_{H B} \\
(\mathrm{~kJ} / \mathrm{mol})\end{array}$ & $-\mathrm{G} / \mathrm{V}$ \\
\hline \multicolumn{9}{|c|}{ Fluoranthene-TCNB } \\
\hline $\mathrm{N}_{1} \cdots \mathrm{H}_{162}-\mathrm{C}_{161}$ & 2.7494 & 0.0054 & 0.0172 & 0.0036 & $\begin{array}{c}- \\
0.0028\end{array}$ & 0.0006 & $\begin{array}{c}- \\
3.6757\end{array}$ & 1.2857 \\
\hline $\mathrm{N}_{2} \cdots \mathrm{H}_{160}-\mathrm{C}_{159}$ & 2.6256 & 0.0071 & 0.0234 & 0.0049 & $\begin{array}{c}- \\
0.0040\end{array}$ & 0.0009 & $\begin{array}{c}- \\
5.2510\end{array}$ & 1.2250 \\
\hline $\begin{array}{c}\mathrm{N}_{12} \cdots \mathrm{H}_{122^{-}} \\
\mathrm{C}_{121}\end{array}$ & 2.5558 & 0.0081 & 0.0260 & 0.0056 & $\begin{array}{c}- \\
0.0047\end{array}$ & 0.0009 & $\begin{array}{c}- \\
6.1699\end{array}$ & 1.1914 \\
\hline $\begin{array}{c}\mathrm{C}_{118}-\mathrm{H}_{18} \cdots \\
\mathrm{N}_{100}\end{array}$ & 3.6469 & 0.0007 & 0.0027 & 0.0005 & $\begin{array}{c}- \\
0.0002\end{array}$ & 0.0003 & $\begin{array}{c}- \\
0.2626\end{array}$ & 2.500 \\
\hline \multicolumn{9}{|c|}{ Fluoranthene-TFP } \\
\hline $\mathrm{F}_{1} \cdots \mathrm{H}_{88}-\mathrm{C}_{87}$ & 3.0695 & 0.0015 & 0.0082 & 0.0014 & $\begin{array}{c}- \\
0.0008\end{array}$ & 0.0006 & $\begin{array}{c}- \\
1.0502\end{array}$ & 1.7500 \\
\hline $\mathrm{F}_{1} \cdots \mathrm{H}_{127}-\mathrm{C}_{126}$ & 2.6731 & 0.0046 & 0.0202 & 0.0040 & $\begin{array}{c}- \\
0.0029\end{array}$ & 0.0011 & $\begin{array}{c}- \\
3.8070\end{array}$ & 1.3793 \\
\hline $\mathrm{F}_{3} \cdots \mathrm{H}_{127}-\mathrm{C}_{126}$ & 2.7696 & 0.0037 & 0.0185 & 0.0034 & $\begin{array}{c}- \\
0.0022\end{array}$ & 0.0012 & $\begin{array}{c}- \\
2.8880\end{array}$ & 1.5455 \\
\hline $\mathrm{F}_{3} \cdots \mathrm{H}_{112}-\mathrm{C}_{111}$ & 2.8445 & 0.0028 & 0.0141 & 0.0025 & $\begin{array}{c}- \\
0.0016\end{array}$ & 0.0009 & $\begin{array}{c}- \\
2.1004\end{array}$ & 1.5625 \\
\hline $\mathrm{N}_{6} \cdots \mathrm{H}_{108}-\mathrm{C}_{107}$ & 2.6648 & 0.0065 & 0.0219 & 0.0045 & $\begin{array}{c}- \\
0.0035\end{array}$ & 0.0010 & $\begin{array}{c}- \\
4.5946\end{array}$ & 1.2857 \\
\hline $\mathrm{N}_{6} \cdots \mathrm{H}_{155^{-}}-\mathrm{C}_{154}$ & 2.6686 & 0.0066 & 0.0222 & 0.0046 & 0.0036 & 0.0010 & 4.7259 & 1.2778 \\
\hline $\mathrm{N}_{6} \cdots \mathrm{H}_{162}-\mathrm{C}_{161}$ & 3.1057 & 0.0027 & 0.0092 & 0.0018 & $\begin{array}{c}- \\
0.0012\end{array}$ & 0.006 & $\begin{array}{c}- \\
1.5753\end{array}$ & 1.500 \\
\hline $\mathrm{F}_{4} \cdots \mathrm{H}_{38}-\mathrm{C}_{37}$ & 2.8174 & 0.0033 & 0.0146 & 0.0027 & $\begin{array}{c}- \\
0.0017\end{array}$ & 0.0010 & $\begin{array}{c}- \\
2.2317\end{array}$ & 1.5882 \\
\hline
\end{tabular}




\begin{tabular}{|c|c|c|c|c|c|c|c|c|}
\hline $\mathrm{F}_{2} \cdots \mathrm{H}_{40}-\mathrm{C}_{39}$ & 2.8957 & 0.0030 & 0.0160 & 0.0030 & 0.0019 & 0.0011 & 2.4942 & 1.7273 \\
\hline $\mathrm{F}_{2} \cdots \mathrm{H}_{38}-\mathrm{C}_{37}$ & 2.8551 & 0.0034 & 0.0180 & 0.0033 & $\begin{array}{c}- \\
0.0021\end{array}$ & 0.0011 & $\begin{array}{c}- \\
2.7568\end{array}$ & 1.5714 \\
\hline $\mathrm{F}_{2} \cdots \mathrm{H}_{62}-\mathrm{C}_{61}$ & 3.7662 & 0.0002 & 0.0018 & 0.0003 & $\begin{array}{c}- \\
0.0001\end{array}$ & 0.0002 & $\begin{array}{c}- \\
0.1312\end{array}$ & 3.000 \\
\hline $\mathrm{N}_{5} \cdots \mathrm{H}_{53}-\mathrm{C}_{52}$ & 2.6770 & 0.0062 & 0.0207 & 0.0043 & $\begin{array}{c}- \\
0.0033\end{array}$ & 0.0010 & $\begin{array}{c}- \\
4.3321\end{array}$ & 1.3030 \\
\hline $\mathrm{N}_{5} \cdots \mathrm{H}_{44}-\mathrm{C}_{43}$ & 2.6947 & 0.0063 & 0.0215 & 0.0044 & $\begin{array}{c}- \\
0.0034\end{array}$ & 0.0010 & $\begin{array}{c}- \\
4.4634\end{array}$ & 1.2941 \\
\hline $\mathrm{N}_{5} \cdots \mathrm{H}_{92}-\mathrm{C}_{91}$ & 2.6166 & 0.0070 & 0.0236 & 0.0049 & $\begin{array}{c}- \\
0.0039\end{array}$ & 0.0010 & $\begin{array}{c}- \\
5.1197\end{array}$ & 1.2564 \\
\hline \multicolumn{9}{|c|}{ Fluoranthene-OFN } \\
\hline $\mathrm{F}_{93} \cdots \mathrm{H}_{104}-\mathrm{C}_{103}$ & 2.6606 & 0.0050 & 0.0244 & 0.0047 & $\begin{array}{c}- \\
0.0033\end{array}$ & 0.0014 & $\begin{array}{c}- \\
4.3321\end{array}$ & 1.4242 \\
\hline $\mathrm{F}_{36} \cdots \mathrm{H}_{104}-\mathrm{C}_{103}$ & 2.8160 & 0.0033 & 0.0162 & 0.0030 & $\begin{array}{c}- \\
0.0019\end{array}$ & 0.0011 & $\begin{array}{c}- \\
2.4942\end{array}$ & 1.5789 \\
\hline $\mathrm{F}_{36} \cdots \mathrm{H}_{78}-\mathrm{C}_{77}$ & 2.6404 & 0.0046 & 0.0214 & 0.0042 & $\begin{array}{c}- \\
0.0030\end{array}$ & 0.0012 & $\begin{array}{c}- \\
3.9382\end{array}$ & 1.4000 \\
\hline $\mathrm{F}_{32} \cdots \mathrm{H}_{54}-\mathrm{C}_{53}$ & 2.9386 & 0.0024 & 0.0127 & 0.0022 & $\begin{array}{c}- \\
0.0013\end{array}$ & 0.0009 & $\begin{array}{c}- \\
1.7066\end{array}$ & 1.6923 \\
\hline $\mathrm{F}_{33} \cdots \mathrm{H}_{54}-\mathrm{C}_{53}$ & 2.7061 & 0.0042 & 0.0186 & 0.0036 & $\begin{array}{c}- \\
0.0025\end{array}$ & 0.0011 & $\begin{array}{c}- \\
3.2819\end{array}$ & 1.4400 \\
\hline $\mathrm{F}_{35} \cdots \mathrm{H}_{65}-\mathrm{C}_{63}$ & 2.6707 & 0.0046 & 0.0213 & 0.0041 & $\begin{array}{c}- \\
0.0029\end{array}$ & 0.0012 & $\begin{array}{c}- \\
3.8070\end{array}$ & 1.4138 \\
\hline $\mathrm{F}_{31} \cdots \mathrm{H}_{65}-\mathrm{C}_{63}$ & 2.8940 & 0.0027 & 0.0129 & 0.0023 & $\begin{array}{c}- \\
0.0014\end{array}$ & 0.0009 & $\begin{array}{c}- \\
1.8379\end{array}$ & 1.6429 \\
\hline $\mathrm{F}_{31} \cdots \mathrm{H}_{75}-\mathrm{C}_{73}$ & 3.0397 & 0.0018 & 0.0100 & 0.0018 & $\begin{array}{c}- \\
0.0010\end{array}$ & 0.0008 & $\begin{array}{c}- \\
1.3128\end{array}$ & 1.8000 \\
\hline
\end{tabular}




\begin{tabular}{cccccccccc}
$\mathrm{F}_{34} \cdots \mathrm{H}_{47}-\mathrm{C}_{15}$ & 2.4358 & 0.0077 & 0.0332 & 0.0071 & & 0.0011 & & 1.1833 \\
& & & & & 0.0060 & & 7.8765 & \\
$\mathrm{~F}_{34} \cdots \mathrm{H}_{121}-\mathrm{C}_{119}$ & 3.1388 & 0.0014 & 0.0075 & 0.0013 & & 0.0006 & - & \\
& & & & & 0.0007 & & 0.9189 & \\
& & & & & & & & \\
$\mathrm{~F}_{94} \cdots \mathrm{H}_{121}-\mathrm{C}_{119}$ & 3.3058 & 0.0012 & 0.0069 & 0.0011 & - & 0.0004 & - & 1.5714 \\
& & & & & 0.0007 & & 0.9189 & \\
\hline
\end{tabular}

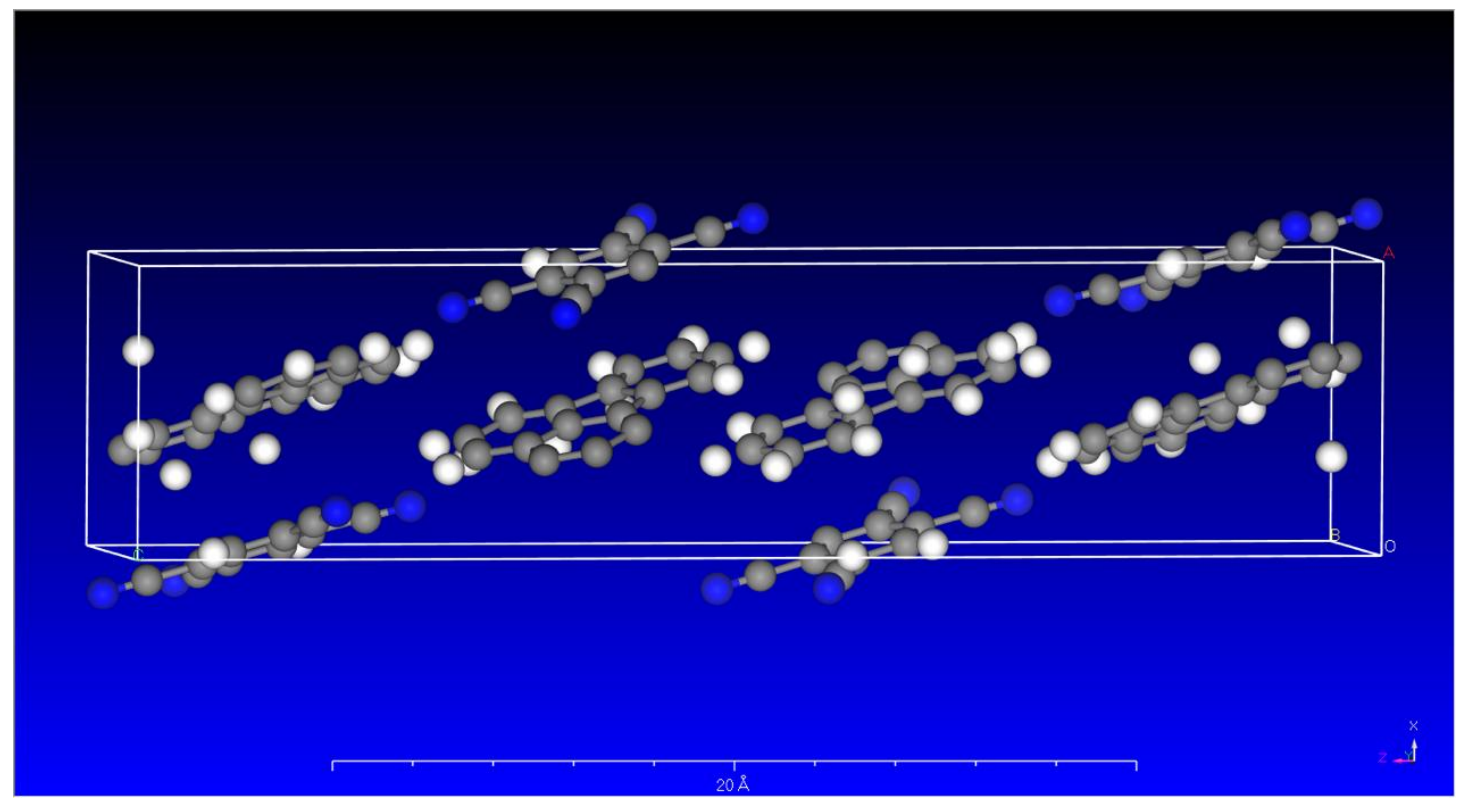

Figure S1. Unit cell of cocrystal of Fluoranthene-TCNB. 


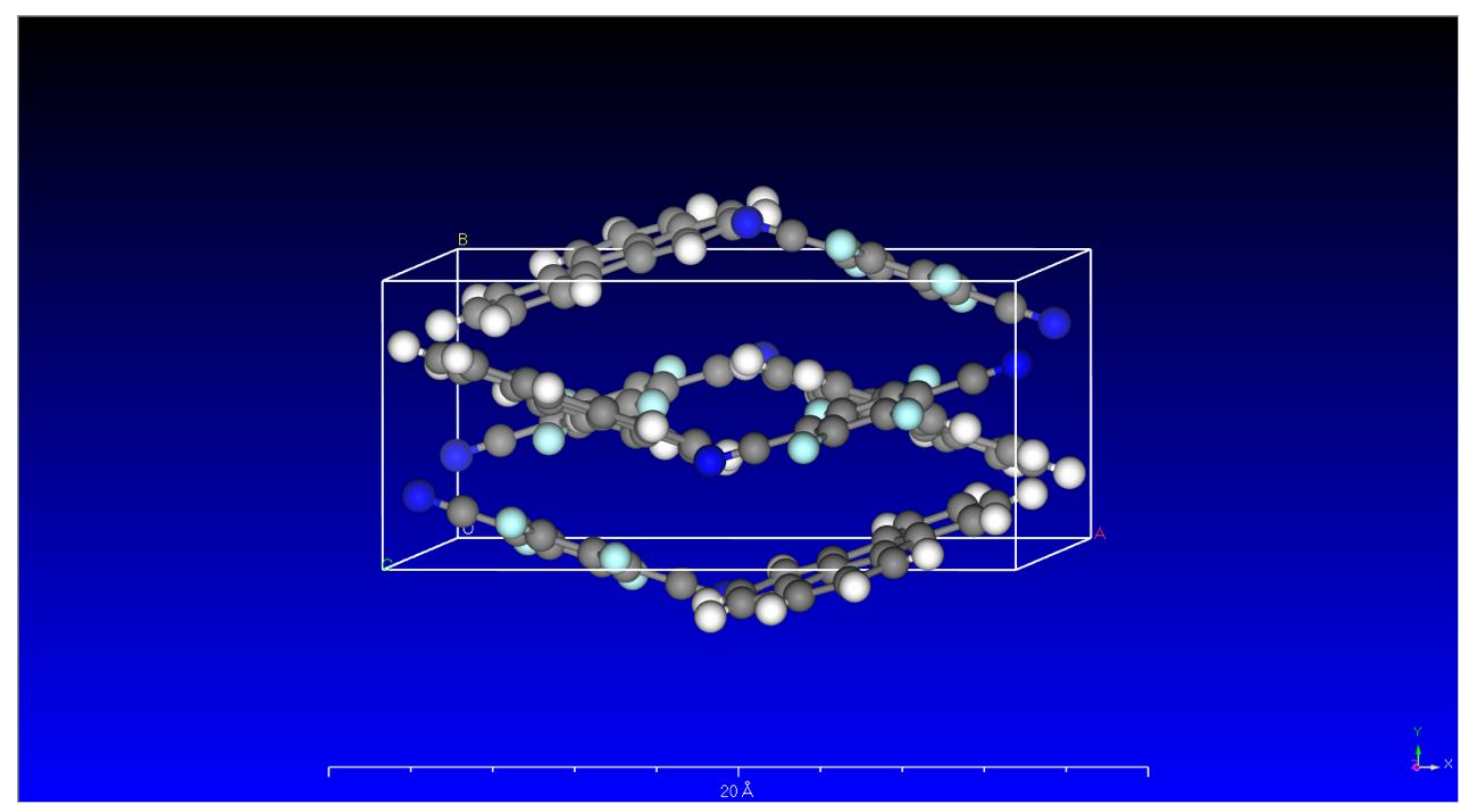

Figure S2. Unit cell of cocrystal of Fluoranthene-TFP. 


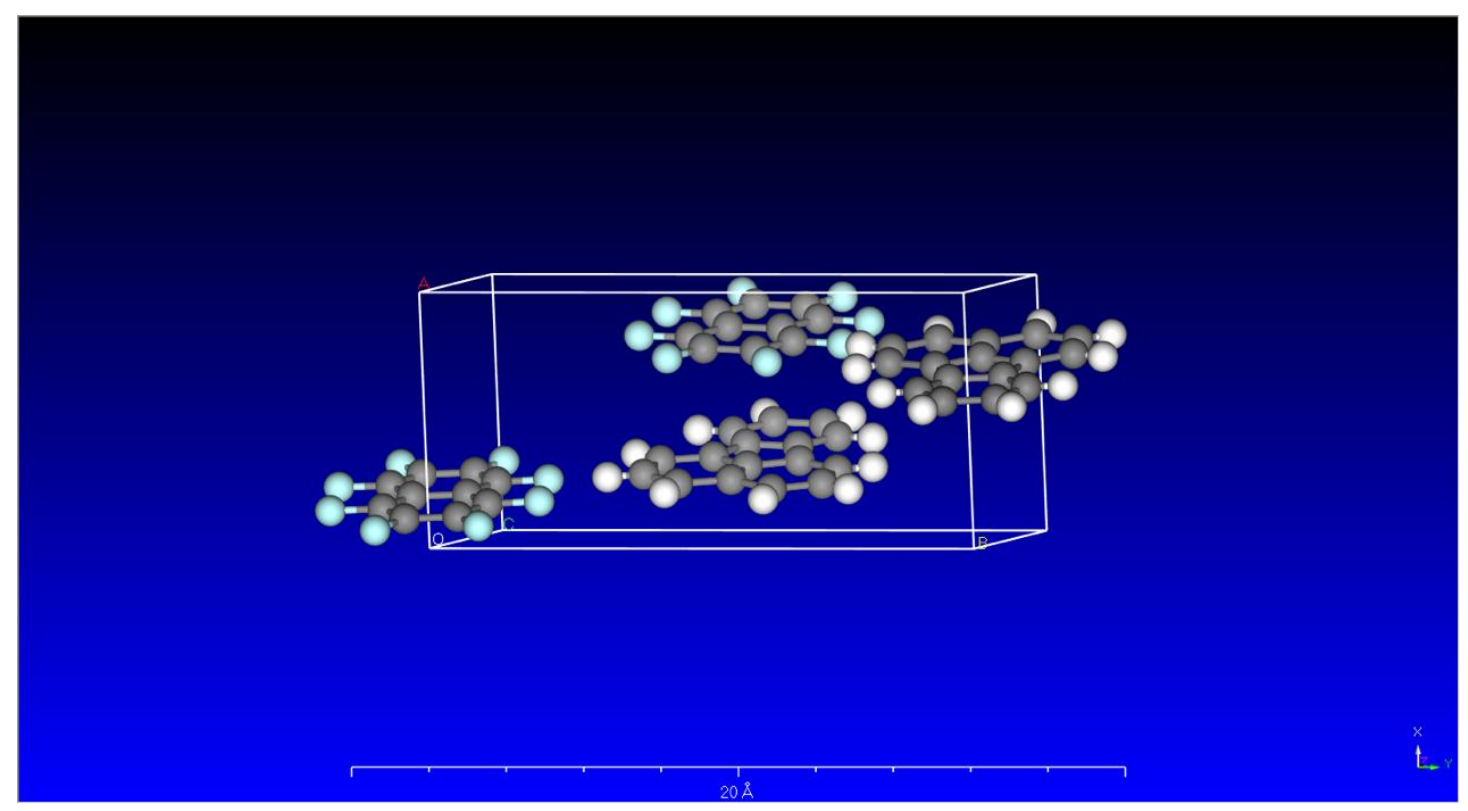

Figure S3. Unit cell of cocrystal of Fluoranthene-OFN. 

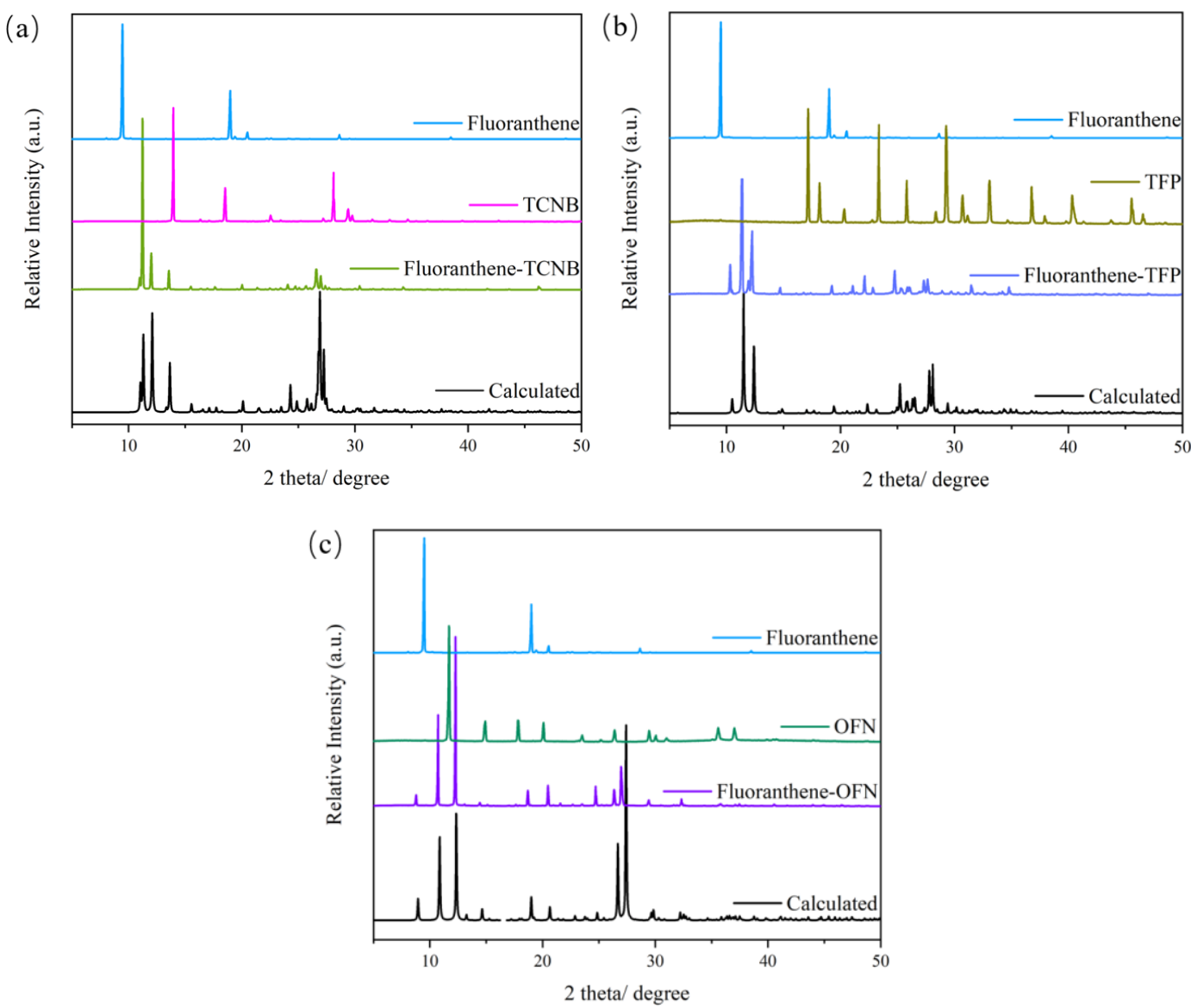

Figure S4. Comparison of the PXRD images of (a) Fluoranthene, TCNB, cocrystal product and calculation values; (b) Fluoranthene, TFP, cocrystal product and calculation values; (c) Fluoranthene, OFN, cocrystal product and calculation values. 


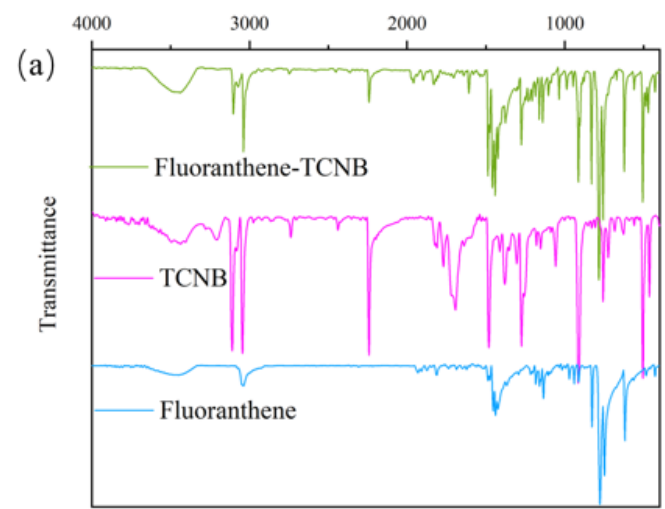

Wavenumber $\left(\mathrm{cm}^{-1}\right)$

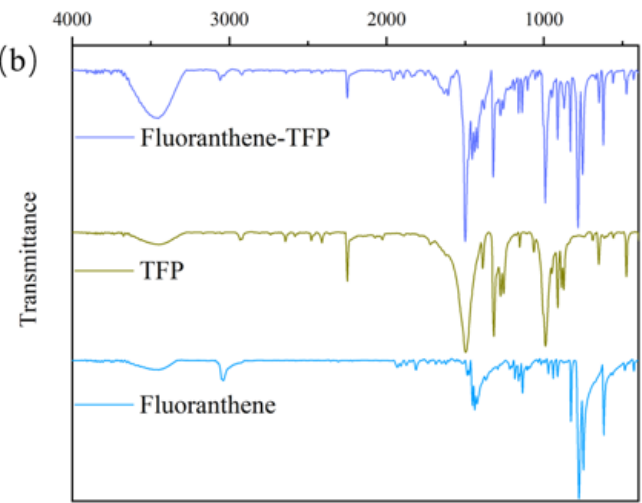

Wavenumber $\left(\mathrm{cm}^{-1}\right)$

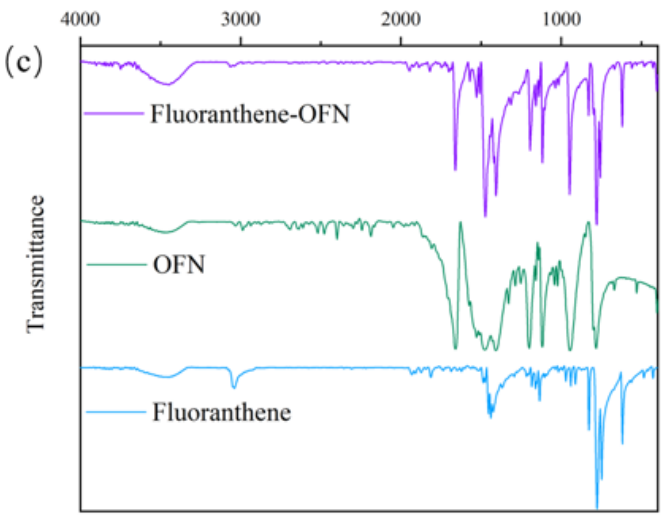

Wavenumber $\left(\mathrm{cm}^{-1}\right)$

Figure S5. IR spectra of (a) Fluoranthene-TCNB; (b) Fluoranthene-TFP; (c) FluorantheneOFN and the co-formers. 

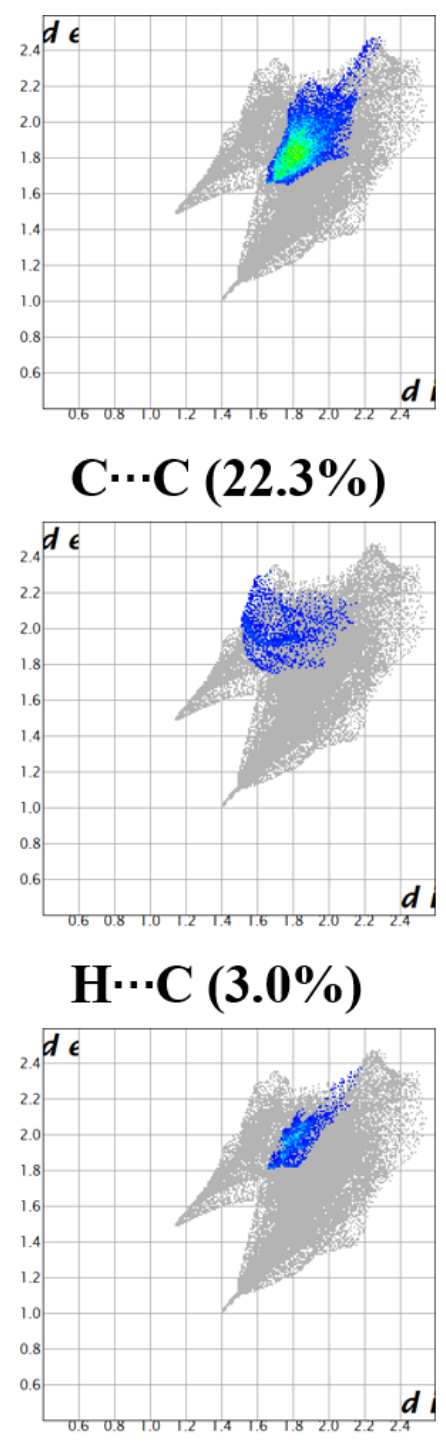

N...C (3.2\%)

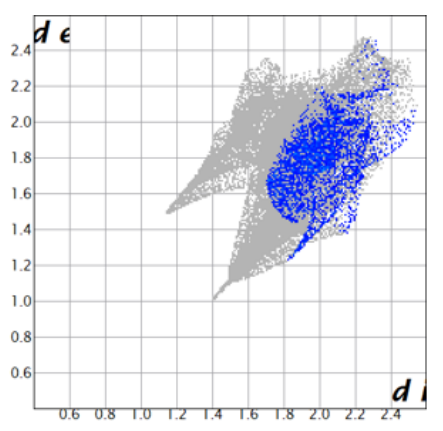

\section{C...H (6.8\%)}
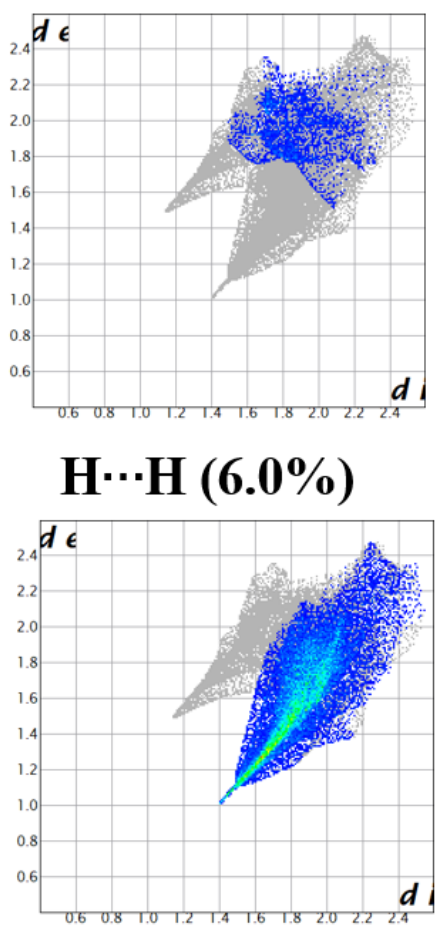

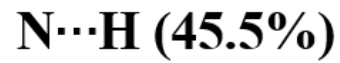

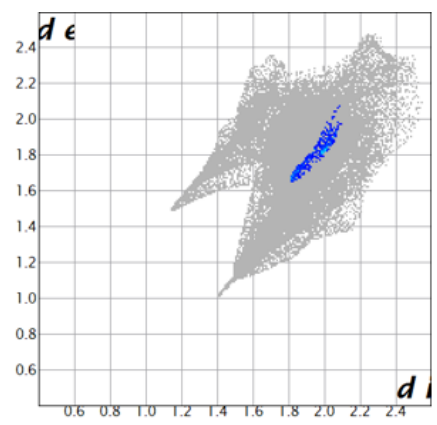

\section{C $\cdots \mathbf{N}(0.8 \%)$}

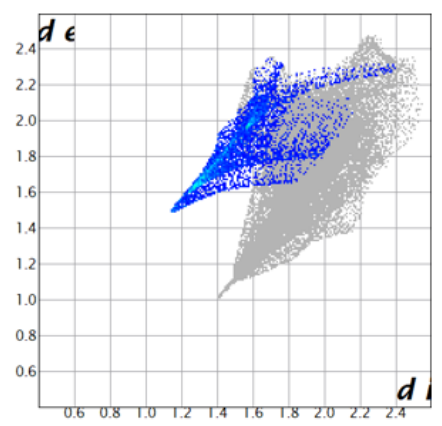

HיN $(8.3 \%)$

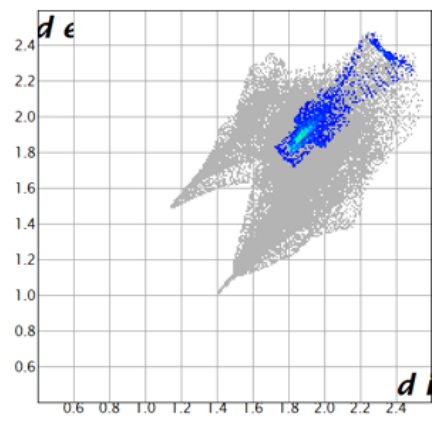

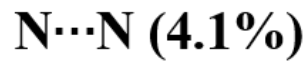

Figure S6. 2-D fingerprint distribution and interaction proportion of Fluoranthene-TCNB. 

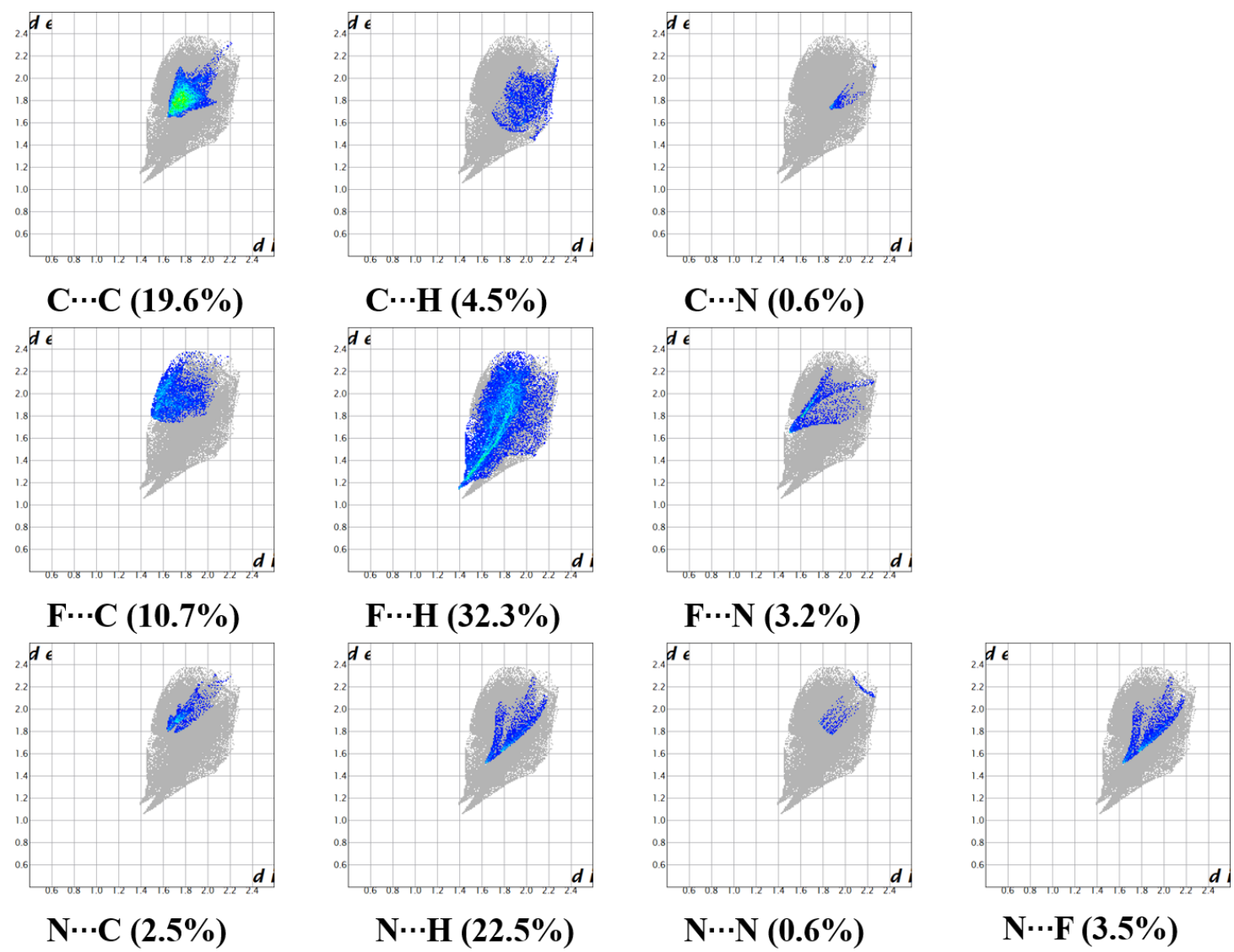

Figure S7. 2-D fingerprint distribution and interaction proportion of Fluoranthene-TFP. 


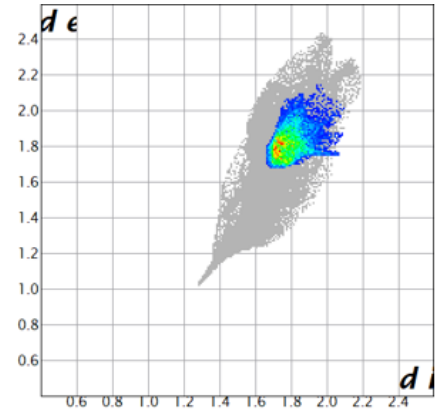

\section{C...C $(23.1 \%)$}

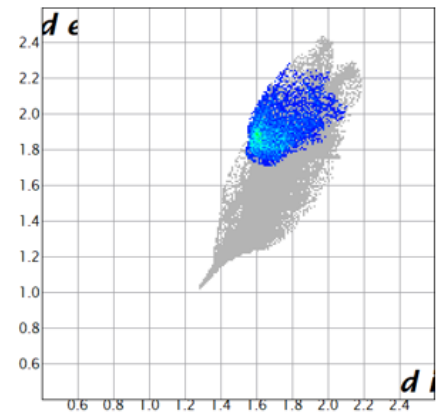

F $\cdots \mathbf{C}(\mathbf{1 0 . 8 \%})$

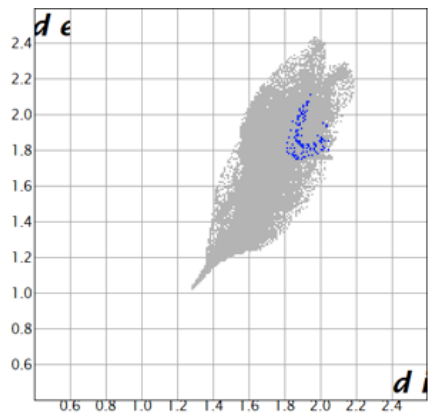

C...H $(0.3 \%)$

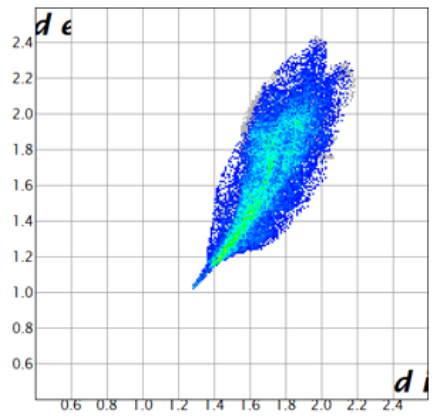

F $\cdots \mathbf{H}(52.6 \%)$

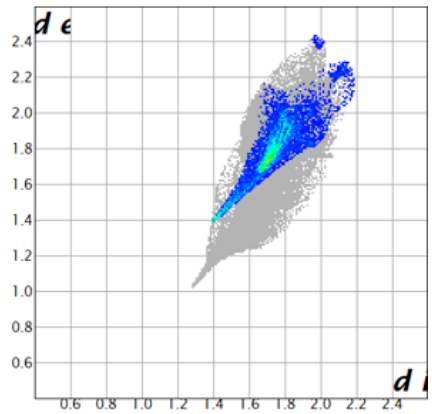

F $\cdots$ F $(13.2 \%)$

Figure S8. 2-D fingerprint distribution and interaction proportion of Fluoranthene-OFN. 